Supporting Information for:

\title{
A conservative algorithm for an adaptive change of resolution in mixed atomistic / coarse-grained multiscale simulations
}

Andreas Heyden and Donald G. Truhlar

To be published in J. Chem. Theory Comput.

Date of preparation of this supplement: January 2, 2008

Total number of pages: 23

\section{Contents}

1. Derivation of the mixed-resolution Hamiltonian .............................................. S-2

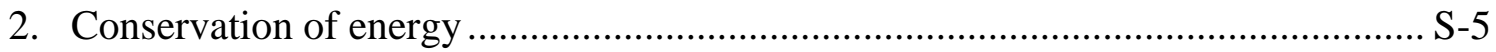

3. Conservation of linear momentum............................................................. S-6

4. Conservation of angular momentum............................................................. S-10

5. Constraints on low-resolution group coordinates and Newton's $3^{\text {rd }}$ law ................ S-16

6. Generalized leapfrog algorithm for mixed-resolution simulations ....................... S-19

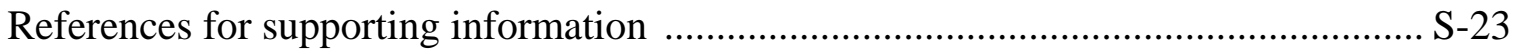




\section{Derivation of the mixed-resolution Hamiltonian}

In this section, we present a detailed derivation of the mixed-resolution Hamiltonian $H^{\mathrm{AP}}$ from the adaptively partitioned Lagrangian function $L^{\mathrm{AP}}$ defined by Eq. 4 of the manuscript, where all symbols are defined in the manuscript. Substituting Eq. 1 into Eq. 4, we obtain

$$
\begin{aligned}
L^{\mathrm{AP}} & =T+\sum_{\beta=1, \cdots, K} S_{\beta}\left(T_{\beta}-T\right)-V^{\mathrm{AP}}=T^{\mathrm{AP}}-V^{\mathrm{AP}} \\
& =\sum_{\alpha=1}^{N} \frac{1}{2} M_{\alpha} \boldsymbol{U}_{\alpha}^{2}+\sum_{k=1}^{A M} \frac{1}{2} m_{k} \boldsymbol{u}_{k}^{2}+\sum_{\beta=1}^{K}\left[\left(1-S_{\beta}\right) \frac{1}{2} M_{\beta} \boldsymbol{U}_{\beta}^{2}+S_{\beta} \sum_{i=1}^{A} \frac{1}{2} m_{i} \boldsymbol{u}_{i}^{2}\right]-V^{\mathrm{AP}}
\end{aligned}
$$

where $T$ is the kinetic energy of the active zone treated on an all-atomistic level and all other groups treated on a low-resolution level, $T_{\beta}$ is the kinetic energy of the active zone and group $\beta$ in the buffer zone treated on an all-atomistic level and all other groups treated on a lowresolution level, and $T^{\mathrm{AP}}$ is the mixed-resolution kinetic energy. Equation S-1 can also be written as

$$
\begin{aligned}
L^{\mathrm{AP}}=\sum_{w=x, y, z}\{ & \sum_{\alpha=1}^{N} \frac{1}{2} M_{\alpha} U_{\alpha, w}^{2}+\sum_{k=1}^{A M} \frac{1}{2} m_{k} u_{k, w}^{2} \\
& \left.+\sum_{\beta=1}^{K}\left[\left(1-S_{\beta}\right) \frac{1}{2} M_{\beta} \boldsymbol{U}_{\beta}^{2}+S_{\beta} \sum_{i=1}^{A} \frac{1}{2} m_{i} \boldsymbol{u}_{i}^{2}\right]\right\}-V^{\mathrm{AP}}
\end{aligned}
$$

The kinetic energy of low-resolution group $\beta$ can be written as 
$\sum_{w=x, y, z} \frac{1}{2} M_{\beta} U_{\beta, w}^{2}$

$$
=\sum_{w=x, y, z} \frac{1}{2}\left(\begin{array}{lll}
u_{1, w}^{(\beta)} & \ldots & u_{A, w}^{(\beta)}
\end{array}\right)\left[\frac{1}{M_{\beta}}\left(\begin{array}{c}
m_{1}^{(\beta)} \\
\vdots \\
m_{A}^{(\beta)}
\end{array}\right)\left(\begin{array}{lll}
m_{1}^{(\beta)} & \ldots & m_{A}^{(\beta)}
\end{array}\right)\right]\left(\begin{array}{c}
u_{1, w}^{(\beta)} \\
\vdots \\
u_{A, w}^{(\beta)}
\end{array}\right)
$$

and Eq. S-2 becomes

$$
\begin{aligned}
& L^{\mathrm{AP}}=\sum_{w=x, y, z}\left\{\sum_{\alpha=1}^{N} \frac{1}{2} M_{\alpha} U_{\alpha, w}^{2}+\sum_{k=1}^{A M} \frac{1}{2} m_{k} u_{k, w}^{2}\right. \\
& +\sum_{\beta=1}^{K}\left[\left(1-S_{\beta}\right) \frac{1}{2}\left(\begin{array}{lll}
u_{1, w}^{(\beta)} & \ldots & u_{A, w}^{(\beta)}
\end{array}\right) \boldsymbol{M}_{D}^{(\beta)}\left(\begin{array}{c}
u_{1, w}^{(\beta)} \\
\vdots \\
u_{A, w}^{(\beta)}
\end{array}\right)\right. \\
& \left.\left.+S_{\beta} \frac{1}{2}\left(\begin{array}{lll}
u_{1, w}^{(\beta)} & \ldots & u_{A, w}^{(\beta)}
\end{array}\right) \boldsymbol{M}_{M}^{(\beta)}\left(\begin{array}{c}
u_{1, w}^{(\beta)} \\
\vdots \\
u_{A, w}^{(\beta)}
\end{array}\right)\right]\right\}-V^{\mathrm{AP}} \\
& =\sum_{w=x, y, z}\left[\sum_{\alpha=1}^{N} \frac{1}{2} M_{\alpha} U_{\alpha, w}^{2}+\sum_{k=1}^{A M} \frac{1}{2} m_{k} u_{k, w}^{2}\right. \\
& \left.+\sum_{\beta=1, \cdots, K} \frac{1}{2}\left(\begin{array}{lll}
u_{1, w}^{(\beta)} & \ldots & u_{A, w}^{(\beta)}
\end{array}\right) \Lambda^{(\beta)}\left(\begin{array}{c}
u_{1, w}^{(\beta)} \\
\vdots \\
u_{A, w}^{(\beta)}
\end{array}\right)\right]-V^{\mathrm{AP}}
\end{aligned}
$$

where

$$
\boldsymbol{\Lambda}^{(\beta)}=\left[\left(1-S_{\beta}\right) \boldsymbol{M}_{D}^{(\beta)}+S_{\beta} \boldsymbol{M}_{M}^{(\beta)}\right]
$$

with 


$$
\boldsymbol{M}_{D}^{(\beta)}=\left[\frac{1}{M_{\beta}}\left(\begin{array}{c}
m_{1}^{(\beta)} \\
\vdots \\
m_{A}^{(\beta)}
\end{array}\right)\left(\begin{array}{lll}
m_{1}^{(\beta)} & \ldots & \left.m_{A}^{(\beta)}\right)
\end{array}\right]\right.
$$

and

$$
\boldsymbol{M}_{M}^{(\beta)}=\left[\begin{array}{ccc}
m_{1}^{(\beta)} & \cdots & 0 \\
\vdots & \ddots & \vdots \\
0 & \cdots & m_{A}^{(\beta)}
\end{array}\right]
$$

where $\boldsymbol{M}_{M}^{(\beta)}$ and $\boldsymbol{M}_{D}^{(\beta)}$ are of dimension $A \times A$. Having defined a mixed-resolution Lagrangian function, we can calculate the generalized momenta $p_{i}$ as $\partial L / \partial u_{i}$, and we can obtain the mixed-resolution Hamiltonian function $H^{\mathrm{AP}}$ given in Eq. 11. The mixed-resolution Hamiltonian $H^{\mathrm{AP}}$ is the sum of the mixed-resolution kinetic energy $T^{\mathrm{AP}}$ and potential energy $V^{\mathrm{AP}}$, and can also be written as

$$
\begin{aligned}
H^{\mathrm{AP}}=H+ & \sum_{\alpha=1, \cdots, K} S_{\alpha}\left(H_{\alpha}-H\right) \\
& +\sum_{\substack{\alpha=1, \cdots, K-1 \\
\beta=\alpha+1, \cdots, K}} S_{\alpha} S_{\beta}\left\{H_{\alpha \beta}-\left[H+\sum_{\mu=\alpha, \beta}\left(H_{\mu}-H\right)\right]\right\} \\
& +\sum_{\substack{\alpha=1, \cdots, K-2 \\
\beta=\alpha+1, \cdots, K-1 \\
\gamma=\beta+1, \cdots, K}} S_{\alpha} S_{\beta} S_{\gamma}\left[H_{\alpha \beta \gamma}-\left(H+\sum_{\mu=\alpha, \beta, \gamma}\left(H_{\mu}-H\right)\right.\right. \\
& \left.+\sum_{\substack{(\sigma, \tau)=(\alpha, \beta),(\alpha, \gamma),(\beta, \gamma) \\
(H)}}\left\{H_{\sigma \tau}-\left[H+\sum_{\mu=\alpha, \beta}\left(H_{\mu}-H\right)\right]\right\}\right]+\cdots
\end{aligned}
$$

which is the Hamiltonian description analogous to Eq. 4 of the manuscript. 


\section{Conservation of energy}

In this section, we show that the mixed-resolution Hamiltonian $H^{\mathrm{AP}}$ conserves energy. Since insertion or removal of atomistic structure on the boundaries of the buffer zone does not alter the kinetic energy, potential energy, or immediate trajectory of the group, the proof that the Hamiltonian conserves energy follows the standard procedure outlined in Goldstein et al. ${ }^{1}$

The mixed-resolution Lagrangian $L^{\mathrm{AP}}$ is not explicitly time dependent. As a result, the total time derivative of the Lagrangian is

$$
\frac{d L^{\mathrm{AP}}}{d t}=\sum_{i=1}^{3 A(K+M+N)} \frac{\partial L^{\mathrm{AP}}}{\partial q_{i}} \dot{q}_{i}+\sum_{i=1}^{3 A(K+M+N)} \frac{\partial L^{\mathrm{AP}}}{\partial \dot{q}_{i}} \ddot{q}_{i}
$$

where the upper limits of the sums are the number of generalized coordinates, $q_{i}$ is a generalized (in this case Cartesian) coordinate $i, \dot{q}_{i}$ is the first time derivative of $q_{i}$, and $\ddot{q}_{i}$ is the second time derivative of $q_{i}$. Lagrange's equation of motion are

$$
\frac{\partial L^{\mathrm{AP}}}{\partial q_{i}}=\frac{d}{d t}\left(\frac{\partial L^{\mathrm{AP}}}{\partial \dot{q}_{i}}\right)
$$

Summing the product of each Lagrange equation with the time derivative of its generalized coordinate

$$
\sum_{i=1}^{3 A(K+M+N)} \frac{\partial L^{\mathrm{AP}}}{\partial q_{i}} \dot{q}_{i}=\sum_{i=1}^{3 A(K+M+N)} \dot{q}_{i} \frac{d}{d t}\left(\frac{\partial L^{\mathrm{AP}}}{\partial \dot{q}_{i}}\right)
$$

and inserting it into Eq. S-9, we find

$$
\frac{d L^{\mathrm{AP}}}{d t}=\sum_{i=1}^{3 A(K+M+N)}\left(\dot{q}_{i} \frac{d}{d t}\left(\frac{\partial L^{\mathrm{AP}}}{\partial \dot{q}_{i}}\right)+\frac{\partial L^{\mathrm{AP}}}{\partial \dot{q}_{i}} \ddot{q}_{i}\right)
$$


Equivalently,

$$
\frac{d L^{\mathrm{AP}}}{d t}=\sum_{i=1}^{3 A(K+M+N)} \frac{d}{d t}\left(\dot{q}_{i} \frac{\partial L^{\mathrm{AP}}}{\partial \dot{q}_{i}}\right)
$$

Finally, we can reorder

$$
0=\frac{d}{d t}\left[\sum_{i=1}^{3 A(K+M+N)} \dot{q}_{i} \frac{\partial L^{\mathrm{AP}}}{\partial \dot{q}_{i}}-L^{A P}\right]=\frac{d H^{\mathrm{AP}}}{d t}
$$

In the preceding section, we showed that the Hamiltonian is the sum of the mixed-resolution kinetic energy $T^{\mathrm{AP}}$ and potential energy $V^{\mathrm{AP}}$ proving that the system energy $\left(E=T^{\mathrm{AP}}+V^{\mathrm{AP}}\right)$ is conserved.

\section{Conservation of linear momentum}

To show that the mixed-resolution Hamiltonian $H^{\mathrm{AP}}$ conserves linear momentum we illustrate that the Poisson bracket $[\cdots, \cdots]$ of the Hamiltonian and the linear momentum in the $x$ direction is zero. The Poisson bracket $[\cdots, \cdots]$ of the Hamiltonian and the linear momentum in the $x$-direction can be written as

$$
\begin{gathered}
{\left[\sum_{j=1}^{3 A(K+M+N)} p_{j, x}, H^{\mathrm{AP}}\right]} \\
\quad=\sum_{\rho=1}^{3 A(K+M+N)}\left(\frac{\partial \sum_{j=1}^{3 A(K+M+N)} p_{j, x}}{\partial q_{\rho}} \frac{\partial H^{\mathrm{AP}}}{\partial p_{\rho}}-\frac{\partial \sum_{j=1}^{3 A(K+M+N)} p_{j, x}}{\partial p_{\rho}} \frac{\partial H^{\mathrm{AP}}}{\partial q_{\rho}}\right)
\end{gathered}
$$




$$
=-\sum_{\rho=1}^{3 A(K+M+N)}\left(\frac{\partial T^{\mathrm{AP}}}{\partial q_{\rho}}+\frac{\partial V^{\mathrm{AP}}}{\partial q_{\rho}}\right)
$$

where $p_{j, x}$ is the generalized momentum of atom $j$ in the $x$-direction, $p_{\rho}$ and $q_{\rho}$ are one of the $3 A(K+M+N)$ generalized momenta and coordinates of the system, respectively, $T^{\mathrm{AP}}$ is the mixed-resolution kinetic energy, and $V^{\mathrm{AP}}$ is the mixed-resolution potential energy. To illustrate that the mixed-resolution Hamiltonian conserves linear momentum we first show that the Poisson bracket of the mixed-resolution potential energy and the linear momentum in the $x$ direction is zero:

$$
\sum_{\rho=1}^{3 A(K+M+N)} \frac{\partial V^{\mathrm{AP}}}{\partial q_{\rho}}=0
$$

Then we show that the Poisson bracket of the mixed-resolution kinetic energy and the linear momentum in the $x$-direction is zero:

$$
\sum_{\rho=1}^{3 A(K+M+N)} \frac{\partial T^{\mathrm{AP}}}{\partial q_{\rho}}=0
$$

The potential energy, $V^{\mathrm{AP}}$, can be written as

$$
V^{\mathrm{AP}}=V+\sum_{\alpha=1, \cdots, K} S_{\alpha} \widetilde{V_{\alpha}}+\sum_{\substack{\alpha=1, \cdots, K-1 \\ \beta=\alpha+1, \cdots, K}} S_{\alpha} S_{\beta} \widetilde{V_{\alpha \beta}}+\sum_{\substack{\alpha=1, \cdots, K-2 \\ \beta=\alpha+1, \cdots, K-1 \\ \gamma=\beta+1, \cdots, K}} S_{\alpha} S_{\beta} S_{\gamma} \widetilde{V_{\alpha \beta \gamma}}+\cdots
$$

where $\widetilde{V_{\mu}} \in\left\{\widetilde{V_{\alpha}}, \widetilde{V_{\alpha \beta}}, \widetilde{V_{\alpha \beta \gamma}}, \ldots\right\}$ is a sum (or difference) of potential energy terms from the set of functions $\left\{V, V_{\alpha}, V_{\alpha \beta}, V_{\alpha \beta \gamma}, \cdots\right\}$ defined in Eq. 4. In the following, we assume that the Hamiltonian conserves linear momentum for a constant set of low-resolution and atomistic groups, i.e., 


$$
\sum_{\rho=1}^{3 A(K+M+N)} \frac{\partial \widetilde{V_{\mu}}}{\partial q_{\rho}}=0
$$

Then it follows that

$$
\begin{aligned}
\sum_{\rho=1}^{3 A(K+M+N)} \frac{\partial V^{\mathrm{AP}}}{\partial q_{\rho}} & =\sum_{\alpha=1, \cdots, K} \widetilde{V_{\alpha}} \sum_{\rho=1}^{3 A(K+M+N)} \frac{\partial S_{\alpha}}{\partial q_{\rho}} \\
& +\sum_{\substack{\alpha=1, \cdots, K-1 \\
\beta=\alpha+1, \cdots, K}} \widetilde{V_{\alpha, \beta}}\left(S_{\alpha} \sum_{\rho=1}^{3 A(K+M+N)} \frac{\partial S_{\beta}}{\partial q_{\rho}}+S_{\beta} \sum_{\rho=1}^{3 A(K+M+N)} \frac{\partial S_{\alpha}}{\partial q_{\rho}}\right) \\
& +\sum_{\substack{\alpha=1, \cdots, K-2 \\
\beta=\alpha+1, \cdots, K-1 \\
\gamma=\beta+1, \cdots, K}} \widetilde{V_{\alpha, \beta, \gamma}}\left(\sum_{\alpha} \sum_{\rho=1}^{3 A(K+M+N)} \frac{\partial S_{\gamma}}{\partial q_{\rho}}+S_{\alpha} S_{\gamma} \sum_{\rho=1}^{3 A(K+M+N)} \frac{\partial S_{\beta}}{\partial q_{\rho}}\right. \\
& \left.+S_{\beta} S_{\gamma} \sum_{\rho=1}^{3 A(K+M+N)} \frac{\partial S_{\alpha}}{\partial q_{\rho}}\right)+\cdots
\end{aligned}
$$

Consequently, a sufficient condition for Eq. S-16 is that for all smoothing functions $S_{\alpha}$,

$$
\sum_{\rho=1}^{3 A(K+M+N)} \frac{\partial S_{\alpha}}{\partial q_{\rho}}=0
$$

This condition is a constraint on the definition of low-resolution sites (groups) and smoothing functions and can be understood as requiring that the Poisson bracket of each smoothing function $S_{\alpha}$ and the linear momentum in the $x$-direction has to be zero. All constraints on the definition of groups are discussed further in Section 5 below. 
In the following we show that the Poisson bracket of the mixed-resolution kinetic energy and the linear momentum in the $x$-direction is zero.

$$
\begin{aligned}
& 3 A(K+M+N) \\
& \sum_{\rho=1}^{(K+M+N)} \frac{\partial T^{\mathrm{AP}}}{\partial q_{\rho}}
\end{aligned}
$$

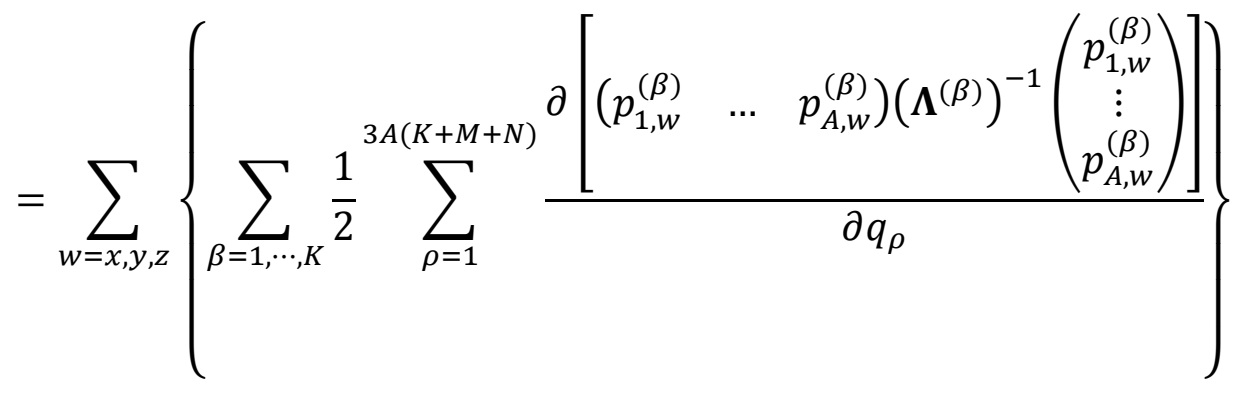

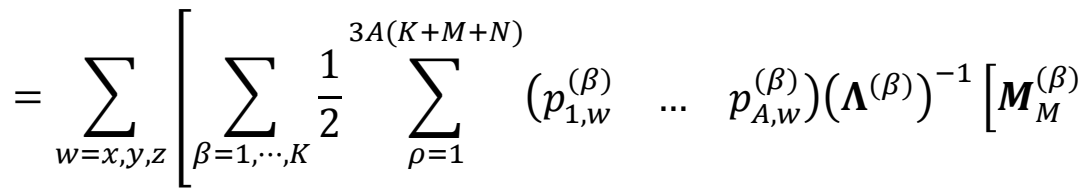

$$
\begin{aligned}
& \left.\left.-\boldsymbol{M}_{D}^{(\beta)}\right]\left(\boldsymbol{\Lambda}^{(\beta)}\right)^{-1}\left(\begin{array}{c}
p_{1, w}^{(\beta)} \\
\vdots \\
p_{A, w}^{(\beta)}
\end{array}\right) \frac{\partial S_{\beta}}{\partial q_{\rho}}\right]
\end{aligned}
$$

After reordering Eq. S-22 we obtain

$$
\begin{aligned}
& \sum_{\rho=1}^{3 A(K+M+N)} \frac{\partial T^{\mathrm{AP}}}{\partial q_{\rho}} \\
& =\sum_{w=x, y, z}\left[\sum _ { \beta = 1 , \cdots , K } \frac { 1 } { 2 } ( p _ { 1 , w } ^ { ( \beta ) } \quad \cdots \quad p _ { A , w } ^ { ( \beta ) } ) ( \boldsymbol { \Lambda } ^ { ( \beta ) } ) ^ { - 1 } \left[\boldsymbol{M}_{M}^{(\beta)}\right.\right. \\
& \left.\left.-\boldsymbol{M}_{D}^{(\beta)}\right]\left(\Lambda^{(\beta)}\right)^{-1}\left(\begin{array}{c}
p_{1, w}^{(\beta)} \\
\vdots \\
p_{A, w}^{(\beta)}
\end{array}\right) \sum_{\rho=1}^{3 A(K+M+N)} \frac{\partial S_{\beta}}{\partial q_{\rho}}\right]
\end{aligned}
$$


Again, a sufficient condition for the Poisson bracket of the mixed-resolution kinetic energy and the linear momentum in the $x$-direction to be zero and therefore also for the mixed-resolution Hamiltonian to conserve linear momentum is Eq. S-21.

\section{Conservation of angular momentum}

To show that the mixed-resolution Hamiltonian $H^{\mathrm{AP}}$ conserves angular momentum we illustrate that the Poisson bracket $[\cdots, \cdots]$ of the total angular momentum in the $x$-direction and that the Hamiltonian, $H^{\mathrm{AP}}=T^{\mathrm{AP}}+V^{\mathrm{AP}}$, is zero. The Poisson bracket of the total angular momentum in the $x$-direction and the Hamiltonian can be written as

$$
\begin{gathered}
{\left[\sum_{j=1}^{3 A(K+M+N)}\left(q_{j, y} p_{j, z}-q_{j, z} p_{j, y}\right), H^{\mathrm{AP}}\right]} \\
=\sum_{\rho=1}^{3 A(K+M+N)}\left(\frac{\partial \sum_{j=1}^{3 A(K+M+N)}\left(q_{j, y} p_{j, z}-q_{j, z} p_{j, y}\right)}{\partial q_{\rho}} \frac{\partial H^{\mathrm{AP}}}{\partial p_{\rho}}\right. \\
\left.-\frac{\partial \sum_{j=1}^{3 A(K+M+N)}\left(q_{j, y} p_{j, z}-q_{j, z} p_{j, y}\right)}{\partial p_{\rho}} \frac{\partial H^{\mathrm{AP}}}{\partial q_{\rho}}\right)
\end{gathered}
$$




$$
\begin{aligned}
=\sum_{j=1}^{3 A(K+M+N)}\left(p_{j, z} \frac{\partial T^{\mathrm{AP}}}{\partial p_{j, y}}-p_{j, y} \frac{\partial T^{\mathrm{AP}}}{\partial p_{j, z}}\right) & \\
& -\sum_{j=1}^{3 A(K+M+N)}\left(q_{j, y} \frac{\partial T^{\mathrm{AP}}}{\partial q_{j, z}}-q_{j, z} \frac{\partial T^{\mathrm{AP}}}{\partial q_{j, y}}\right) \\
& -\sum_{j=1}^{3 A(K+M+N)}\left(q_{j, y} \frac{\partial V^{\mathrm{AP}}}{\partial q_{j, z}}-q_{j, z} \frac{\partial V^{\mathrm{AP}}}{\partial q_{j, y}}\right)
\end{aligned}
$$

where $p_{j, y}, p_{j, z}, q_{j, y}$, and $q_{j, z}$ are the generalized momenta and coordinates of atom $j$ in the $y$ and z-direction, respectively. To illustrate that the mixed-resolution Hamiltonian conserves angular momentum in the $x$-direction we show that the first two sums in Eq. S-25 are both zero

$$
\sum_{j=1}^{3 A(K+M+N)}\left(p_{j, z} \frac{\partial T^{\mathrm{AP}}}{\partial p_{j, y}}-p_{j, y} \frac{\partial T^{\mathrm{AP}}}{\partial p_{j, z}}\right)=0
$$

and

$$
\sum_{j=1}^{3 A(K+M+N)}\left(q_{j, y} \frac{\partial T^{\mathrm{AP}}}{\partial q_{j, z}}-q_{j, z} \frac{\partial T^{\mathrm{AP}}}{\partial q_{j, y}}\right)=0
$$

i.e., that the Poisson bracket of the mixed-resolution kinetic energy and the angular momentum in the $x$-direction is zero. Next we show that the Poisson bracket of the mixed-resolution potential energy and the angular momentum in the $x$-direction is zero.

$$
\sum_{j=1}^{3 A(K+M+N)}\left(q_{j, y} \frac{\partial V^{\mathrm{AP}}}{\partial q_{j, z}}-q_{j, z} \frac{\partial V^{\mathrm{AP}}}{\partial q_{j, y}}\right)=0
$$


The mixed-resolution kinetic energy can be written as

$$
T^{\mathrm{AP}}=\sum_{w=x, y, z}\left[\sum_{\alpha=1}^{N} \frac{P_{\alpha, w}^{2}}{2 M_{\alpha}}+\sum_{k=1}^{A M} \frac{p_{k, w}^{2}}{2 m_{k}}+\sum_{\beta=1, \cdots, K} \frac{1}{2}\left(\begin{array}{lll}
p_{1, w}^{(\beta)} & \cdots & p_{A, w}^{(\beta)}
\end{array}\right)\left(\Lambda^{(\beta)}\right)^{-1}\left(\begin{array}{c}
p_{1, w}^{(\beta)} \\
\vdots \\
p_{A, w}^{(\beta)}
\end{array}\right)\right]
$$

so that

$$
\begin{aligned}
& \sum_{j=1}^{3 A(K+M+N)}\left(p_{j, z} \frac{\partial T^{\mathrm{AP}}}{\partial p_{j, y}}-p_{j, y} \frac{\partial T^{\mathrm{AP}}}{\partial p_{j, z}}\right) \\
& =\sum_{\alpha=1}^{N}\left[\sum_{k=1}^{A}\left(p_{k, z} \frac{P_{\alpha, y}}{M_{\alpha}}-p_{k, y} \frac{P_{\alpha, z}}{M_{\alpha}}\right)\right]+\sum_{k=1}^{A M}\left(p_{k, z} \frac{p_{k, y}}{m_{k}}-p_{k, y} \frac{p_{k, z}}{m_{k}}\right) \\
& +\sum_{\beta=1, \cdots, \mathrm{K}}\left[\begin{array}{lll}
\left(p_{1, Z}^{(\beta)}\right. & \cdots & p_{A, Z}^{(\beta)}
\end{array}\right)\left(\Lambda^{(\beta)}\right)^{-1}\left(\begin{array}{c}
p_{1, y}^{(\beta)} \\
\vdots \\
p_{A, y}^{(\beta)}
\end{array}\right) \\
& \left.-\left(\begin{array}{lll}
p_{1, y}^{(\beta)} & \ldots & p_{A, y}^{(\beta)}
\end{array}\right)\left(\Lambda^{(\beta)}\right)^{-1}\left(\begin{array}{c}
p_{1, z}^{(\beta)} \\
\vdots \\
p_{A, z}^{(\beta)}
\end{array}\right)\right] \\
& =\sum_{\alpha=1}^{N}\left(P_{\alpha, z} \frac{P_{\alpha, y}}{M_{\alpha}}-P_{\alpha, y} \frac{P_{\alpha, z}}{M_{\alpha}}\right) \\
& +\sum_{\beta=1, \cdots, \mathrm{K}}\left[\begin{array}{lll}
\left(p_{1, Z}^{(\beta)}\right. & \cdots & p_{A, z}^{(\beta)}
\end{array}\right)\left(\Lambda^{(\beta)}\right)^{-1}\left(\begin{array}{c}
p_{1, y}^{(\beta)} \\
\vdots \\
p_{A, y}^{(\beta)}
\end{array}\right) \\
& \left.-\left(\begin{array}{lll}
p_{1, y}^{(\beta)} & \ldots & p_{A, y}^{(\beta)}
\end{array}\right)\left(\Lambda^{(\beta)}\right)^{-\mathrm{T}}\left(\begin{array}{c}
p_{1, z}^{(\beta)} \\
\vdots \\
p_{A, z}^{(\beta)}
\end{array}\right)\right]=0
\end{aligned}
$$


where $\left(\Lambda^{(\beta)}\right)^{-1}$ is symmetric. As a result, a sufficient condition for the Poisson bracket of the kinetic energy and the angular momentum in the $x$-direction to be zero is Eq. S-26. The left hand side of Eq. S-26 can be written as

$$
\begin{aligned}
& \sum_{j=1}^{3 A(K+M+N)}\left(q_{j, y} \frac{\partial T^{\mathrm{AP}}}{\partial q_{j, z}}-q_{j, z} \frac{\partial T^{\mathrm{AP}}}{\partial q_{j, y}}\right) \\
& =\sum_{j=1}^{3 A(K+M+N)}\left(q _ { j , y } \left[\sum _ { w = x , y , z } \sum _ { \beta = 1 , \cdots , K } \left(-\frac{1}{2}\left(p_{1, w}^{(\beta)} \quad \ldots \quad p_{A, w}^{(\beta)}\right)\left(\boldsymbol{\Lambda}^{(\beta)}\right)^{-1}\left[\boldsymbol{M}_{M}^{(\beta)}\right.\right.\right.\right. \\
& \left.\left.\left.-\boldsymbol{M}_{D}^{(\beta)}\right]\left(\boldsymbol{\Lambda}^{(\beta)}\right)^{-1}\left(\begin{array}{c}
p_{1, w}^{(\beta)} \\
\vdots \\
p_{A, w}^{(\beta)}
\end{array}\right) \frac{\partial S_{\beta}}{\partial q_{j, z}}\right)\right] \\
& -q_{j, z}\left[\sum _ { w = x , y , z } \sum _ { \beta = 1 , \cdots , K } ( \begin{array} { l l l } 
{ - \frac { 1 } { 2 } ( p _ { 1 , w } ^ { ( \beta ) } } & { \cdots } & { p _ { A , w } ^ { ( \beta ) } }
\end{array} ) ( \boldsymbol { \Lambda } ^ { ( \beta ) } ) ^ { - 1 } \left[\boldsymbol{M}_{M}^{(\beta)}\right.\right. \\
& \left.\left.\left.\left.-\boldsymbol{M}_{D}^{(\beta)}\right]\left(\boldsymbol{\Lambda}^{(\beta)}\right)^{-1}\left(\begin{array}{c}
p_{1, w}^{(\beta)} \\
\vdots \\
p_{A, w}^{(\beta)}
\end{array}\right) \frac{\partial S_{\beta}}{\partial q_{j, y}}\right)\right]\right) \\
& =\sum_{w=x, y, z} \sum_{\beta=1, \cdots, K}\left(-\frac{1}{2}\left(\begin{array}{lll}
p_{1, w}^{(\beta)} & \ldots & p_{A, w}^{(\beta)}
\end{array}\right)\left(\boldsymbol{\Lambda}^{(\beta)}\right)^{-1}\left[\boldsymbol{M}_{M}^{(\beta)}\right.\right. \\
& \left.\left.-\boldsymbol{M}_{D}^{(\beta)}\right]\left(\boldsymbol{\Lambda}^{(\beta)}\right)^{-1}\left(\begin{array}{c}
p_{1, w}^{(\beta)} \\
\vdots \\
p_{A, w}^{(\beta)}
\end{array}\right) \sum_{j=1}^{3 A(K+M+N)}\left[q_{j, y} \frac{\partial S_{\beta}}{\partial q_{j, z}}-q_{j, z} \frac{\partial S_{\beta}}{\partial q_{j, y}}\right]\right)
\end{aligned}
$$

Consequently, a sufficient condition for Eq. S-26 is that for all smoothing functions $S_{\beta}$, 


$$
\sum_{j=1}^{3 A(K+M+N)}\left[q_{j, y} \frac{\partial S_{\beta}}{\partial q_{j, z}}-q_{j, z} \frac{\partial S_{\beta}}{\partial q_{j, y}}\right]=0
$$

This condition is a constraint on the definition of low-resolution sites (groups) and smoothing functions and can be understood as requiring that the Poisson bracket of each smoothing function $S_{\beta}$ and the angular momentum in the $x$-direction has to be zero. This constraint on the definition of groups is discussed further in Section 5 below.

Finally, we illustrate that the Poisson bracket of the mixed-resolution potential energy and the angular momentum in the $x$-direction is zero, i.e., Eq. S-27 is fulfilled. The mixed-resolution potential energy, $V^{\mathrm{AP}}$, can be written as

$$
V^{\mathrm{AP}}=V+\sum_{\alpha=1, \cdots, K} S_{\alpha} \widetilde{V_{\alpha}}+\sum_{\substack{\alpha=1, \cdots, K-1 \\ \beta=\alpha+1, \cdots, K}} S_{\alpha} S_{\beta} \widetilde{V_{\alpha \beta}}+\sum_{\substack{\alpha=1, \cdots, K-2 \\ \beta=\alpha+1, \cdots, K-1 \\ \gamma=\beta+1, \cdots, K}} S_{\alpha} S_{\beta} S_{\gamma} \widetilde{V_{\alpha \beta \gamma}}+\cdots
$$

where $\widetilde{V_{\mu}} \in\left\{\widetilde{V_{\alpha}}, \widetilde{V_{\alpha \beta}}, \widetilde{V_{\alpha \beta \gamma}}, \ldots\right\}$ is a sum (or difference) of potential energy terms from the set of functions $\left\{V, V_{\alpha}, V_{\alpha \beta}, V_{\alpha \beta \gamma}, \cdots\right\}$ defined in Eq. 4. In the following, we assume that the Hamiltonian conserves angular momentum for a constant set of low-resolution and atomistic groups, i.e.,

$$
\sum_{j=1}^{3 A(K+M+N)}\left(q_{j, y} \frac{\partial \widetilde{V}_{\mu}}{\partial q_{j, z}}-q_{j, z} \frac{\partial \widetilde{V}_{\mu}}{\partial q_{j, y}}\right)=0
$$

It follows that 


$$
\begin{aligned}
& \sum_{j=1}^{3 A(K+M+N)}\left[q_{j, y} \frac{\partial V^{\mathrm{AP}}}{\partial q_{j, z}}-q_{j, z} \frac{\partial V^{\mathrm{AP}}}{\partial q_{j, y}}\right] \\
& =\sum_{\alpha=1, \cdots, K} \widetilde{V}_{\alpha} \sum_{j=1}^{3 A(K+M+N)}\left[q_{j, y} \frac{\partial S_{\alpha}}{\partial q_{j, z}}-q_{j, z} \frac{\partial S_{\alpha}}{\partial q_{j, y}}\right] \\
& +\sum_{\substack{\alpha=1, \cdots, K-1 \\
\beta=\alpha+1, \cdots, K}} \widetilde{V_{\alpha, \beta}}\left(S_{\alpha} \sum_{j=1}^{3 A(K+M+N)}\left[q_{j, y} \frac{\partial S_{\beta}}{\partial q_{j, z}}-q_{j, z} \frac{\partial S_{\beta}}{\partial q_{j, y}}\right]\right. \\
& \left.+S_{\beta} \sum_{j=1}^{3 A(K+M+N)}\left[q_{j, y} \frac{\partial S_{\alpha}}{\partial q_{j, z}}-q_{j, z} \frac{\partial S_{\alpha}}{\partial q_{j, y}}\right]\right) \\
& +\sum_{\substack{\alpha=1, \cdots, K-2 \\
\beta=\alpha+1, \cdots, K-1 \\
\gamma=\beta+1, \cdots, K}} \widetilde{V_{\alpha, \beta, \gamma}}\left(S_{\alpha} S_{\beta} \sum_{j=1}^{3 A(K+M+N)}\left[q_{j, y} \frac{\partial S_{\gamma}}{\partial q_{j, z}}-q_{j, z} \frac{\partial S_{\gamma}}{\partial q_{j, y}}\right]\right. \\
& +S_{\alpha} S_{\gamma} \sum_{j=1}^{3 A(K+M+N)}\left[q_{j, y} \frac{\partial S_{\beta}}{\partial q_{j, z}}-q_{j, z} \frac{\partial S_{\beta}}{\partial q_{j, y}}\right] \\
& \left.+S_{\beta} S_{\gamma} \sum_{j=1}^{3 A(K+M+N)}\left[q_{j, y} \frac{\partial S_{\alpha}}{\partial q_{j, z}}-q_{j, z} \frac{\partial S_{\alpha}}{\partial q_{j, y}}\right]\right)+\cdots
\end{aligned}
$$

Again, a sufficient condition for the Poisson bracket of the potential energy and the angular momentum in the $x$-direction to be zero and therefore also for the mixed-resolution Hamiltonian to conserve angular momentum is Eq. S-31. 


\section{Constraints on low-resolution group coordinates and Newton's $3^{\text {rd }}$ law}

Equations S-21 and S-31 set a number of constraints on the definition of low-resolution groups. In particular, these equations require that the Poisson bracket of the linear and angular momentum with all smoothing function $S_{\alpha}$ is zero. In the following, we will first show that Eqs. S-21 and S-31 are fulfilled for the placement of the center of a group on a specific atom. Then we will show that Eqs. S-21 and S-31 are fulfilled for the placement of the center of a group on the center of mass of the group. Without loss of generality we will illustrate both possibilities by assuming that the center of an active site is placed on an atom $k$. In the course

of illustrating the fulfillment of Eq. S-21 we also show that Newton's $3^{\text {rd }}$ law is fulfilled for the APL Hamiltonian.

\subsection{Center of group is considered to be on an atom.}

\subsubsection{Constraints enforced by conservation of linear momentum}

$$
\begin{gathered}
\sum_{\rho=1}^{3 A(K+M+N)} \frac{\partial S_{\alpha}}{\partial q_{\rho}}=\sum_{\rho=1}^{3 A(K+M+N)} \frac{\partial S_{\alpha}}{\partial \Omega_{\alpha}} \frac{\partial \Omega_{\alpha}}{\partial q_{\rho}} \\
=\frac{\partial S_{\alpha}}{\partial \Omega_{\alpha}} \frac{1}{r_{\max }-r_{\min }} \sum_{\rho=1}^{3 A(K+M+N)} \frac{\partial r_{\alpha, k}}{\partial q_{\rho}} \\
=\frac{\partial S_{\alpha}}{\partial \Omega_{\alpha}} \frac{1}{r_{\max }-r_{\min }} \frac{1}{r_{\alpha, k}}\left[\left(q_{k, x}-q_{\alpha, x}\right)+\left(q_{k, y}-q_{\alpha, y}\right)+\left(q_{k, z}-q_{\alpha, z}\right)\right. \\
\left.\quad-\left[\left(q_{k, x}-q_{\alpha, x}\right)+\left(q_{k, y}-q_{\alpha, y}\right)+\left(q_{k, z}-q_{\alpha, z}\right)\right]\right]
\end{gathered}
$$

$=0$ 
where $r_{\alpha, k}$ is the distance between the low-resolution site $\alpha$ and the active site $k, q_{k, x}, q_{k, y}, q_{k, z}$ are the Cartesian coordinates of the atom representing the center of the active site, and $q_{\alpha, x}$, $q_{\alpha, y}, q_{\alpha, z}$ are the Cartesian coordinates of the atom defining the position of group $\alpha$. Following the derivation in Eq. S-35, it can be seen that Newton's $3^{\text {rd }}$ law (for every action, there is an equal and opposite reaction) is fulfilled if the mixed-resolution potential energy functional $V^{(\mu, v)}$ in Eq. 1 fulfills Newton's $3^{\text {rd }}$ law for a fixed number of coarse-grained and atomistic sites. We note that for common coarse-grained and atomistic potential energy functions and a mixed-resolution potential energy functional of form Eq. 2, Newton's $3^{\text {rd }}$ law is therefore fulfilled.

\subsubsection{Constraints enforced by conservation of angular momentum}

$$
\begin{gathered}
\sum_{j=1}^{3 A(K+M+N)}\left[q_{\rho, y} \frac{\partial S_{\alpha}}{\partial q_{\rho, z}}-q_{\rho, z} \frac{\partial S_{\alpha}}{\partial q_{\rho, y}}\right] \\
=\frac{\partial S_{\alpha}}{\partial \Omega_{\alpha}} \frac{1}{r_{\max }-r_{\min }} \sum_{\rho=1}^{3 A(K+M+N)}\left[q_{\rho, y} \frac{\partial r_{\alpha, k}}{\partial q_{\rho, z}}-q_{\rho, z} \frac{\partial r_{\alpha, k}}{\partial q_{\rho, y}}\right] \\
=\frac{\partial S_{\alpha}}{\partial \Omega_{\alpha}} \frac{1}{r_{\max }-r_{\min }} \frac{1}{r_{\alpha, k}}\left[q_{k, y}\left(q_{k, z}-q_{\alpha, z}\right)-q_{k, z}\left(q_{k, y}-q_{\alpha, y}\right)\right. \\
\left.+q_{\alpha, y}\left(q_{\alpha, z}-q_{k, z}\right)-q_{\alpha, z}\left(q_{\alpha, y}-q_{k, y}\right)\right] \\
=0
\end{gathered}
$$




\subsection{Center of group is considered to be at the center of mass.}

\subsubsection{Constraints enforced by conservation of linear momentum}

$$
\begin{gathered}
\sum_{\rho=1}^{3 A(K+M+N)} \frac{\partial S_{\alpha}}{\partial q_{\rho}}=\sum_{\rho=1}^{3 A(K+M+N)} \frac{\partial S_{\alpha}}{\partial \Omega_{\alpha}} \frac{\partial \Omega_{\alpha}}{\partial q_{\rho}} \\
=\frac{\partial S_{\alpha}}{\partial \Omega_{\alpha}} \frac{1}{r_{\max }-r_{\min }} \sum_{\rho=1}^{3 A(K+M+N)} \frac{\partial r_{\alpha, k}}{\partial q_{\rho}} \\
=\frac{\partial S_{\alpha}}{\partial \Omega_{\alpha}} \frac{1}{r_{\max }-r_{\min }} \frac{1}{r_{\alpha, k}}\left[\left(q_{k, x}-q_{\alpha, x}\right)+\left(q_{k, y}-q_{\alpha, y}\right)+\left(q_{k, z}-q_{\alpha, z}\right)\right. \\
\quad-\left\{\sum _ { i = 1 } ^ { A } \frac { m _ { i } ^ { ( \alpha ) } } { M _ { \alpha } } \left[\left(q_{k, x}-q_{i, x}^{(\alpha)}\right)+\left(q_{k, y}-q_{i, y}^{(\alpha)}\right)\right.\right. \\
\left.\left.\left.\quad+\left(q_{k, z}-q_{i, z}^{(\alpha)}\right)\right]\right\}\right] \\
=0
\end{gathered}
$$

where $M_{\alpha}$ is the mass of group $\alpha$ that is composed of $A$ atoms each of mass $m_{i}^{(\alpha)}$ and $q_{\alpha, x}=\sum_{i=1}^{A} \frac{m_{i}^{(\alpha)}}{M_{\alpha}} q_{i, x}^{(\alpha)}$ etc. Following the derivation in Eq. S-37, it can be seen that Newton's $3^{\text {rd }}$ law is fulfilled on a group basis if the mixed-resolution potential energy functional $V^{(\mu, v)}$ in Eq. 1 fulfills Newton's $3^{\text {rd }}$ law for a fixed number of coarse-grained and atomistic sites. We note again that for common coarse-grained and atomistic potential energy functions and a mixed-resolution potential energy functional of form Eq. 2, Newton's $3^{\text {rd }}$ law is therefore fulfilled. 


\subsubsection{Constraints enforced by conservation of angular momentum}

$$
\begin{gathered}
\sum_{\rho=1}^{3 A(K+M+N)}\left[q_{\rho, y} \frac{\partial S_{\alpha}}{\partial q_{\rho, z}}-q_{\rho, z} \frac{\partial S_{\alpha}}{\partial q_{\rho, y}}\right] \\
=\frac{\partial S_{\alpha}}{\partial \Omega_{\alpha}} \frac{1}{r_{\max }-r_{\min }} \sum_{\rho=1}^{3 A(K+M+N)}\left[q_{\rho, y} \frac{\partial r_{\alpha, k}}{\partial q_{\rho, z}}-q_{\rho, z} \frac{\partial r_{\alpha, k}}{\partial q_{\rho, y}}\right] \\
=\frac{\partial S_{\alpha}}{\partial \Omega_{\alpha}} \frac{1}{r_{\max }-r_{\min }} \frac{1}{r_{\alpha, k}}\left[q_{k, y}\left(q_{k, z}-q_{\alpha, z}\right)-q_{k, z}\left(q_{k, y}-q_{\alpha, y}\right)\right. \\
+\sum_{i=1}^{A} \frac{m_{i}^{(\alpha)}}{M_{\alpha}} q_{i, y}^{(\alpha)}\left(q_{i, z}^{(\alpha)}-q_{k, z}\right) \\
\left.\quad-\sum_{i=1}^{A} \frac{m_{i}^{(\alpha)}}{M_{\alpha}} q_{i, z}^{(\alpha)}\left(q_{i, y}^{(\alpha)}-q_{k, y}\right)\right] \\
=0
\end{gathered}
$$

6. Generalized leapfrog algorithm for mixed-resolution simulations

The generalized leapfrog method ${ }^{2}$ is given by Eqs. S-39 to S-41.

$$
\begin{gathered}
\boldsymbol{p}^{\left(n+\frac{1}{2}\right)}=\boldsymbol{p}^{(n)}-\frac{\Delta \mathrm{t}}{2} \boldsymbol{\nabla}_{q} H\left(\boldsymbol{p}^{\left(n+\frac{1}{2}\right)}, \boldsymbol{q}^{(n)}\right) \\
\boldsymbol{q}^{(n+1)=} \boldsymbol{q}^{(n)}+\frac{\Delta \mathrm{t}}{2}\left[\boldsymbol{\nabla}_{p} H\left(\boldsymbol{p}^{\left(n+\frac{1}{2}\right)}, \boldsymbol{q}^{(n)}\right)+\nabla_{p} H\left(\boldsymbol{p}^{\left(n+\frac{1}{2}\right)}, \boldsymbol{q}^{(n+1)}\right)\right] \\
\boldsymbol{p}^{(n+1)}=\boldsymbol{p}^{\left(n+\frac{1}{2}\right)}-\frac{\Delta \mathrm{t}}{2} \boldsymbol{\nabla}_{q} H\left(\boldsymbol{p}^{\left(n+\frac{1}{2}\right)}, \boldsymbol{q}^{(n+1)}\right)
\end{gathered}
$$


where $n, n+1 / 2$, and $n+1$ denote a time step. The mixed-resolution Hamiltonian $H^{\mathrm{AP}}$ is given by

$$
\begin{aligned}
H^{\mathrm{AP}}=\sum_{w=x, y, z} & {\left[\sum_{\alpha=1}^{N} \frac{P_{\alpha, w}^{2}}{2 M_{\alpha}}+\sum_{k=1}^{A M} \frac{p_{k, w}^{2}}{2 m_{k}}+\sum_{\beta=1, \cdots, K} \frac{1}{2}\left(\begin{array}{lll}
p_{1, w}^{(\beta)} & \cdots & p_{A, w}^{(\beta)}
\end{array}\right)\left(\Lambda^{(\beta)}\right)^{-1}\left(\begin{array}{c}
p_{1, w}^{(\beta)} \\
\vdots \\
p_{A, w}^{(\beta)}
\end{array}\right)\right] } \\
& +V^{\mathrm{AP}}
\end{aligned}
$$

The derivative of the Hamiltonian with respect to coordinate $q_{k, v}, v \in\{x, y, z\}$ is:

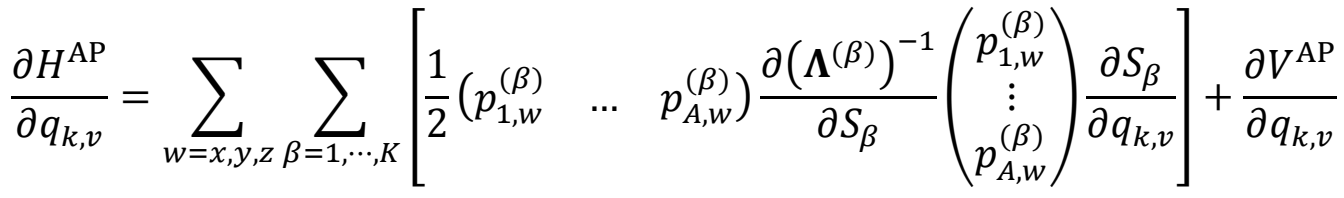

$$
\begin{aligned}
& =\sum_{w=x, y, z} \sum_{\beta=1, \cdots, K}\left[\begin{array}{lll}
-\frac{1}{2}\left(p_{1, w}^{(\beta)}\right. & \cdots & \left.p_{A, w}^{(\beta)}\right)\left(\boldsymbol{\Lambda}^{(\beta)}\right)^{-1}\left[\boldsymbol{M}_{M}^{(\beta)}\right.
\end{array}\right. \\
& \left.\left.-\boldsymbol{M}_{D}^{(\beta)}\right]\left(\boldsymbol{\Lambda}^{(\beta)}\right)^{-1}\left(\begin{array}{c}
p_{1, w}^{(\beta)} \\
\vdots \\
p_{A, w}^{(\beta)}
\end{array}\right) \frac{\partial S_{\beta}}{\partial q_{k, v}}\right]+\frac{\partial V^{\mathrm{AP}}}{\partial q_{k, v}}
\end{aligned}
$$

The derivative of the Hamiltonian with respect to the generalized momentum $p_{k, v}$, for atom $k$ being in the active zone, is:

$$
\frac{\partial H^{\mathrm{AP}}}{\partial p_{k, v}}=\frac{p_{k, v}}{m_{k}}
$$

The derivative of the Hamiltonian with respect to the generalized momentum $p_{j, v}^{(\alpha)}$ for atom $j$ of group $\alpha$ being in the environmental zone, is:

$$
\frac{\partial H^{\mathrm{AP}}}{\partial p_{j, v}^{(\alpha)}}=\frac{P_{\alpha, v}}{M_{\alpha}}
$$


where $P_{\alpha, v}$ is the center of mass momentum of group $\alpha$ with mass $M_{\alpha}$. The derivative of the Hamiltonian with respect to the generalized momenta $p_{i, v}^{(\beta)} \forall i \in\{1, \cdots, A\}$ of group $\beta$ being in the buffer zone, is:

$$
\left(\begin{array}{c}
\frac{\partial H^{\mathrm{AP}}}{\partial p_{1, v}^{(\beta)}} \\
\vdots \\
\frac{\partial H^{\mathrm{AP}}}{\partial p_{A, v}^{(\beta)}}
\end{array}\right)=\left(\Lambda^{(\beta)}\right)^{-1}\left(\begin{array}{c}
p_{1, v}^{(\beta)} \\
\vdots \\
p_{A, v}^{(\beta)}
\end{array}\right)
$$

As a result, Eq. S-39 for atom $k$ belonging to group $\alpha$ becomes:

$$
\begin{aligned}
& p_{k, v}^{(n+1 / 2)}=p_{k, v}^{(n)}-\frac{\Delta \mathrm{t}}{2} \frac{\partial V^{\mathrm{AP}, n}}{\partial q_{k, v}} \\
& +\frac{\Delta \mathrm{t}}{4} \sum_{w=x, y, z} \sum_{\beta=1, \cdots, K}\left[( \begin{array} { l l l } 
{ p _ { 1 , w } ^ { ( \beta ) } } & { \cdots } & { p _ { A , w } ^ { ( \beta ) } }
\end{array} ) ^ { ( n + \frac { 1 } { 2 } ) } ( \boldsymbol { \Lambda } ^ { ( \beta , n ) } ) ^ { - 1 } \left[\boldsymbol{M}_{M}^{(\beta)}\right.\right. \\
& \left.\left.-\boldsymbol{M}_{D}^{(\beta)}\right]\left(\boldsymbol{\Lambda}^{(\beta, n)}\right)^{-1}\left(\begin{array}{c}
p_{1, w}^{(\beta)} \\
\vdots \\
p_{A, w}^{(\beta)}
\end{array}\right)^{\left(n+\frac{1}{2}\right)} \frac{\partial S_{\beta}^{(n)}}{\partial q_{k, v}}\right]
\end{aligned}
$$

Eq. S-47 can be solved by a fixed point iteration ${ }^{2}$ for $p_{k, v}^{(n+1 / 2)}$ with $p_{k, v, \text { initial }}^{(n+1 / 2)} \leftarrow p_{k, v}^{(n)}$. (Note: The mixed-resolution potential energy $V^{\mathrm{AP}, n}$ is only calculated once per iteration.) Equation S40 for the atoms of group $\alpha$ becomes:

$$
\left(\begin{array}{c}
q_{1, v} \\
\vdots \\
q_{A, v}
\end{array}\right)^{(n+1)}=\left(\begin{array}{c}
q_{1, v} \\
\vdots \\
q_{A, v}
\end{array}\right)^{(n)}+\frac{\Delta \mathrm{t}}{2}\left[\left(\Lambda^{(\alpha, n)}\right)^{-1}\left(\begin{array}{c}
p_{1, v}^{(\alpha)} \\
\vdots \\
p_{A, v}^{(\alpha)}
\end{array}\right)^{\left(n+\frac{1}{2}\right)}+\left(\Lambda^{(\alpha, n+1)}\right)^{-1}\left(\begin{array}{c}
p_{1, v}^{(\alpha)} \\
\vdots \\
p_{A, v}^{(\alpha)}
\end{array}\right)^{\left(n+\frac{1}{2}\right)}\right]
$$

Equation S-48 can be solved by a second fixed point iteration for the generalized coordinates, $q_{k, v}^{(n+1)}$, with $q_{k, v, \text { initial }}^{(n+1)} \leftarrow q_{k, v}^{(n)}$. (Note: The mixed-resolution potential energy $V^{\mathrm{AP}}$ is not 
calculated during the iteration.) For groups that are in the environmental zone at time step $n$ or $n+1$, the velocity of each atom is equivalent to the center of mass velocity

$$
\left(\Lambda^{(\alpha, n+1)}\right)^{-1}\left(\begin{array}{c}
p_{1, v}^{(\alpha)} \\
\vdots \\
p_{A, v}^{(\alpha)}
\end{array}\right)^{\left(n+\frac{1}{2}\right)}=\frac{P_{\alpha, v}^{\left(n+\frac{1}{2}\right)}}{M_{\alpha}}\left(\begin{array}{c}
1 \\
\vdots \\
1
\end{array}\right)
$$

We point out that Eq. S-48 reduces to the standard explicit leapfrog algorithm for groups in the active zone and environmental zone (at time steps $n$ and $n+1$ ):

$$
q_{k, v}^{(n+1)}=q_{k, v}^{(n)}+\Delta \mathrm{t} \frac{p_{k, v}^{\left(n+\frac{1}{2}\right)}}{m_{k}}
$$

Finally, Eq. S-41 for atom $k$ belonging to group $\alpha$ becomes

$$
\begin{aligned}
& p_{k, v}^{(n+1)}=p_{k, v}^{(n+1 / 2)}-\frac{\Delta \mathrm{t}}{2} \frac{\partial V^{\mathrm{AP}, n+1}}{\partial q_{k, v}}
\end{aligned}
$$

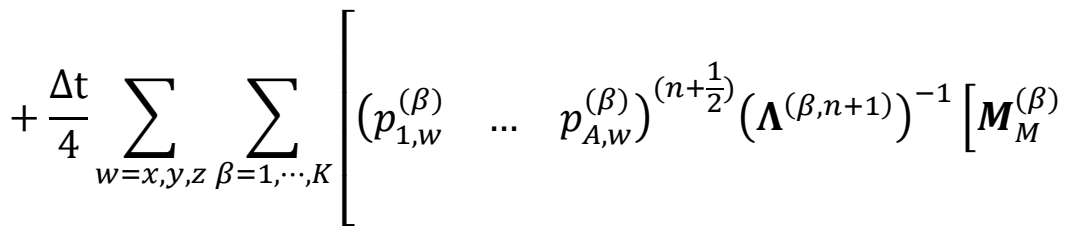

$$
\begin{aligned}
& \left.\left.-\boldsymbol{M}_{D}^{(\beta)}\right]\left(\boldsymbol{\Lambda}^{(\beta, n+1)}\right)^{-1}\left(\begin{array}{c}
p_{1, w}^{(\beta)} \\
\vdots \\
p_{A, w}^{(\beta)}
\end{array}\right)^{\left(n+\frac{1}{2}\right)} \frac{\partial S_{\beta}^{(n+1)}}{\partial q_{k, v}}\right]
\end{aligned}
$$

Equation S-51 is explicit. We note that in the APL scheme the dynamics of the system depends explicitly on both the smoothing function and its derivative. 


\section{References}

(1) Goldstein, H.; Poole, C. P.; Safko, J. L. Classical mechanics, 3rd ed.; Addison Wesley: San Francisco, 2002.

(2) Leimkuhler, B.; Reich, S. Simulating Hamiltonian dynamics; Cambridge University Press: New York, 2004. 